\title{
Parton transport and hadronization from the dynamical quasiparticle point of view
}

\author{
W. Cassing $^{a}$ and E. L. Bratkovskaya ${ }^{b}$ \\ ${ }^{a}$ Institut für Theoretische Physik, Universität Giessen, 35392 Giessen, Germany \\ ${ }^{b}$ Frankfurt Institute for Advanced Studies, JWG Universität Frankfurt, 60438 Frankfurt am Main, Germany
}

\begin{abstract}
The hadronization of an expanding partonic fireball is studied within the Parton-Hadron-Strings Dynamics (PHSD) approach which is based on a dynamical quasiparticle model (DQPM) matched to reproduce lattice QCD results in thermodynamic equilibrium. Apart from strong parton interactions the expansion and development of collective flow is driven by strong gradients in the parton mean-fields. An analysis of the elliptic flow $v_{2}$ demonstrates a linear correlation with the spatial eccentricity $\epsilon$ as in case of ideal hydrodynamics. The hadronization occurs by quark-antiquark fusion or 3 quark/3 antiquark recombination which is described by covariant transition rates. Since the dynamical quarks become very massive, the formed resonant 'pre-hadronic' color-dipole states $(q \bar{q}$ or $q q q)$ are of high invariant mass, too, and sequentially decay to the groundstate meson and baryon octets increasing the total entropy. This solves the entropy problem in hadronization in a natural way. The resulting particle ratios turn out to be in line with those from a grandcanonical partition function at temperature $T \approx 170 \mathrm{MeV}$ rather independent from the initial temperature and indicate an approximate strangeness equilibration.
\end{abstract}

PACS numbers: 25.75.-q, 13.60.Le, 14.40.Lb, 14.65.Dw

\section{INTRODUCTION}

The 'Big Bang' scenario implies that in the first microseconds of the universe the entire state has emerged from a partonic system of quarks, antiquarks and gluons - a quark-gluon plasma (QGP) - to color neutral hadronic matter consisting of interacting hadronic states (and resonances) in which the partonic degrees of freedom are confined. The nature of confinement and the dynamics of this phase transition has motivated a large community for several decades and is still an outstanding question of todays physics. Early concepts of the QGP were guided by the idea of a weakly interacting system of partons which might be described by perturbative QCD (pQCD). However, experimental observations at the Relativistic Heavy Ion Collider (RHIC) indicated that the new medium created in ultrarelativistic $\mathrm{Au}+\mathrm{Au}$ collisions was interacting more strongly than hadronic matter (cf. [1] and Refs. therein) and consequently this concept had to be given up. Moreover, in line with theoretical studies in Refs. [2-4] the medium showed phenomena of an almost perfect liquid of partons $[5,6]$ as extracted from the strong radial expansion and elliptic flow of hadrons [5].

The question about the properties of this (nonperturbative) QGP liquid is discussed controversially in the literature and dynamical concepts describing the formation of color neutral hadrons from partons are scarce [7-13]. A fundamental issue for hadronization models is the conservation of 4-momentum as well as the entropy problem because by fusion/coalescence of massless (or low constituent mass) partons to color neutral bound states of low invariant mass (e.g. pions) the number of degrees of freedom and thus the total entropy is reduced in the hadronization process [9-11]. This problem - a violation of the second law of thermodynamics as well as of the conservation 4-momentum and flavor currents - definitely needs a sound dynamical solution.

A consistent dynamical approach - valid also for strongly interacting systems - can be formulated on the basis of Kadanoff-Baym (KB) equations [14, 15] or offshell transport equations in phase-space representation, respectively [15-17]. In the $\mathrm{KB}$ theory the field quanta are described in terms of propagators with complex selfenergies. Whereas the real part of the selfenergies can be related to mean-field potentials, the imaginary parts provide information about the lifetime and/or reaction rates of time-like 'particles' [4]. Once the proper (complex) selfenergies of the degrees of freedom are known the time evolution of the system is fully governed by off-shell transport equations (as described in Refs. [15-17]).

The determination/extraction of complex selfenergies for the partonic degrees of freedom has been performed in Refs. $[4,18,19]$ by fitting lattice QCD (1QCD) 'data' within the Dynamical QuasiParticle Model (DQPM). In fact, the DQPM allows for a simple and transparent interpretation of lattice QCD results for thermodynamic quantities as well as correlators and leads to effective strongly interacting partonic quasiparticles with broad spectral functions. We stress that mean-field potentials for the 'quarks' and 'gluons' as well as effective interactions have been extracted from lQCD within the DQPM as well (cf. Ref. [19]).

\section{THE PHSD APPROACH}

The Parton-Hadron-String-Dynamics (PHSD) approach is a microscopic covariant transport model that incorporates effective partonic as well as hadronic degrees of freedom and involves a dynamical description of the hadronization process from partonic to hadronic matter. Whereas the hadronic part is essentially equivalent to the 
conventional Hadron-Strings-Dynamics (HSD) approach [20] the partonic dynamics is based on the Dynamical QuasiParticle Model (DQPM) [18, 19] which describes QCD properties in terms of single-particle Green's functions (in the sense of a two-particle irredicible (2PI) approach).

\section{A. Reminder of the DQPM}

We briefly recall the basic assumptions of the DQPM: Following Ref. [21] the dynamical quasiparticle mass (for gluons and quarks) is assumed to be given by the thermal mass in the asymptotic high-momentum regime, which is proportional to the coupling $g\left(T / T_{c}\right)$ and the temperature $T$ with a running coupling (squared),

$$
g^{2}\left(T / T_{c}\right)=\frac{48 \pi^{2}}{\left(11 N_{c}-2 N_{f}\right) \ln \left(\lambda^{2}\left(T / T_{c}-T_{s} / T_{c}\right)^{2}\right.} .
$$

Here $N_{c}=3$ stands for the number of colors while $N_{f}$ denotes the number of flavors. The parameters controlling the infrared enhancement of the coupling $\lambda=2.42$ and $T_{s}=0.46 T_{c}$ have been fitted in [21] to lQCD results for the entropy density $s(T)$. An almost perfect reproduction of the energy density $\varepsilon(T)$ and the pressure $P(T)$ from lQCD is achieved as well. As demonstrated in Fig. 1 of Ref. [19] this functional form for the strong coupling $\alpha_{s}=g^{2} /(4 \pi)$ is in full accordance with the 1QCD calculations of Ref. [22] for the long range part of the $q-\bar{q}$ potential, too.

The width for gluons and quarks (for vanishing chemical potential $\mu_{q}$ ) is adopted in the form [23]

$$
\gamma_{g}(T)=\frac{3 g^{2} T}{8 \pi} \ln \left(\frac{2 c}{g^{2}}\right), \gamma_{q}(T)=\frac{g^{2} T}{6 \pi} \ln \left(\frac{2 c}{g^{2}}\right),
$$

where $c=14.4$ (from Ref. [4]) is related to a magnetic cut-off. We stress that a non-vanishing width $\gamma$ is the central difference of the DQPM to conventional quasiparticle models [24-26]. It influence is essentially seen in correlation functions as e.g. in the stationary limit of the correlation function in the off-diagonal elements of the energy-momentum tensor $T^{k l}$ which defines the shear viscosity $\eta$ of the medium $[4,27]$. Here a sizable width is mandatory to obtain a small ratio in the shear viscosity to entropy density $\eta / s$.

In line with [21] the parton spectral functions thus are no longer $\delta$ - functions in the invariant mass squared but taken as

$$
\rho_{j}(\omega)=\frac{\gamma_{j}}{E_{j}}\left(\frac{1}{\left(\omega-E_{j}\right)^{2}+\gamma_{j}^{2}}-\frac{1}{\left(\omega+E_{j}\right)^{2}+\gamma_{j}^{2}}\right)
$$

separately for quarks and gluons $(j=q, \bar{q}, g)$. With the convention $E^{2}(\boldsymbol{p})=\boldsymbol{p}^{2}+M_{j}^{2}-\gamma_{j}^{2}$, the parameters $M_{j}^{2}$ and $\gamma_{j}$ are directly related to the real and imaginary parts of the retarded self-energy, e.g. $\Pi_{j}=M_{j}^{2}-2 i \gamma_{j} \omega$.
With the spectral functions fixed by Eqs. (1)-(3) the total energy density in the DQPM (at vanishing quark chemical potential) can be evaluated as

$$
\begin{aligned}
T^{00} & =d_{g} \int_{0}^{\infty} \frac{d \omega}{2 \pi} \int \frac{d^{3} p}{(2 \pi)^{3}} 2 \omega^{2} \rho_{g}(\omega, \mathbf{p}) n_{B}(\omega / T) \\
& +d_{q} \int_{0}^{\infty} \frac{d \omega}{2 \pi} \int \frac{d^{3} p}{(2 \pi)^{3}} 2 \omega^{2} \rho_{q}(\omega, \mathbf{p}) n_{F}(\omega / T)
\end{aligned}
$$

where $n_{B}$ and $n_{F}$ denote the Bose and Fermi functions, respectively. The number of transverse gluonic degrees of freedom is $d_{g}=16$ while the fermic degrees of freedom amount to $d_{q}=4 N_{c} N_{f}=36$ in case of three flavors $\left(N_{f}=3\right)$. The pressure $P$ then may be obtained by integrating the differential thermodynamic relation

$$
P-T \frac{\partial P}{\partial T}=-T^{00}
$$

with the entropy density $s$ given by

$$
s=\frac{\partial P}{\partial T}=\frac{T^{00}+P}{T} .
$$

This approach is thermodynamically consistent and represents a 2PI approximation to hot QCD (once the free parameters in (1) and (2) are fitted to lattice QCD results as in Refs. $[4,18,19])$.

As outlined in detail in Refs. [18, 19] the energy density functional (4) can be separated in space-like and time-like sectors when the spectral functions aquire a finite width. The space-like part of (4) defines a potential energy density $V_{p}$ since the field quanta involved are virtuell and correspond to partons exchanged in interaction diagrams. The time-like part of (4) corresponds to effective field quanta which can be propagated within the light-cone. Related separations can be made for virtuell and time-like parton densities $[18,19]$. Without repeating the details we mention that mean-field potentials for partons can be defined by the derivative of the potential energy density $V_{p}$ with respect to the time-like parton densities and effective interactions by second derivatives of $V_{p}$ (cf. Section 3 in Ref. [19]).

\section{B. Hadronization in PHSD}

Based on the DQPM we have developed an off-shell transport approach denoted as PHSD where the degreesof-freedom are dynamical quarks, antiquarks and gluons $(q, \bar{q}, g)$ with rather large masses and broad spectral functions in line with (1) - (3) as well as the conventional hadrons (described in the standard HSD approach [20]). On the partonic side the following elastic and inelastic interactions are included $q q \leftrightarrow q q, \bar{q} \bar{q} \leftrightarrow \bar{q} \bar{q}, g g \leftrightarrow g g$, $g g \leftrightarrow g, q \bar{q} \leftrightarrow g$ exploiting 'detailed-balance' with interaction rates from the DQPM $[4,18,19]$ (cf. Section IIIB). The hadronisation, i.e. transition from partonic to 
hadronic degrees of freedom, is described by local covariant transition rates e.g. for $q+\bar{q}$ fusion to a meson $m$ of four-momentum $p=(\omega, \mathbf{p})$ at space time point $x=(t, \mathbf{x})$ :

$$
\begin{aligned}
& \frac{d N_{m}(x, p)}{d^{4} x d^{4} p}=\operatorname{Tr}_{q} \operatorname{Tr}_{\bar{q}} \delta^{4}\left(p-p_{q}-p_{\bar{q}}\right) \delta^{4}\left(\frac{x_{q}+x_{\bar{q}}}{2}-x\right) \\
& \quad \times \omega_{q} \rho_{q}\left(p_{q}\right) \omega_{\bar{q}} \rho_{\bar{q}}\left(p_{\bar{q}}\right)\left|v_{q \bar{q}}\right|^{2} W_{m}\left(x_{q}-x_{\bar{q}}, p_{q}-p_{\bar{q}}\right) \\
& \quad \times N_{q}\left(x_{q}, p_{q}\right) N_{\bar{q}}\left(x_{\bar{q}}, p_{\bar{q}}\right) \delta \text { (flavor, color). }
\end{aligned}
$$

In (7) we have introduced the shorthand notation

$$
T r_{j}=\sum_{j} \int d^{4} x_{j} d^{4} p_{j} /(2 \pi)^{4}
$$

where $\sum_{j}$ denotes a summation over discrete quantum numbers (spin, flavor, color); $N_{j}(x, p)$ is the phase-space density of parton $j$ at space-time position $x$ and fourmomentum $p$. In Eq. (7) $\delta$ (flavor, color) stands symbolically for the conservation of flavor quantum numbers as well as color neutrality of the formed hadron $m$ which can be viewed as a color-dipole or 'pre-hadron'. Furthermore, $v_{q \bar{q}}\left(\rho_{p}\right)$ is the effective quark-antiquark interaction from the DQPM defined by Eq. (31) and displayed in Fig. 10 of Ref. [19] as a function of the local parton $(q+\bar{q}+g)$ density $\rho_{p}$ (or energy density). Furthermore, $W_{m}(x, p)$ is the phase-space distribution of the formed 'pre-hadron'. It is taken as a Gaussian in coordinate and momentum space (following Ref. [28]) with width $\sqrt{\left\langle r^{2}\right\rangle}=0.66$ $\mathrm{fm}$ (in the rest frame) which corresponds to an average rms radius of mesons. The width in momentum space is fixed by the uncertainty principle, i.e. $\Delta x \Delta p=1$ (in natural units). We note that the final hadron formation rates are approximately independent on these parameters within reasonable variations.

In principle, the two-particle Greens-function $G^{<}\left(x_{q}, p_{q}, x_{\bar{q}}, p_{\bar{q}}\right)$ should appear in Eq. (7). The approximation of the two-particle Greens function by a (symmetrized/antisymmetrized) product of singleparticle Greens functions is always a first step in a cluster expansion for Greens functions and neglects 'residual correlations' stemming from higher order contractions. The same holds for an approximation of the three-particle Greens function by the (symmetrized/antisymmetrized) product of single-particle Greens functions (cf. Ref. [29]). However, the DQPM with its dynamical spectral functions already includes the effects of strong two-body correlations - contrary to bare Green functions - such that the effect of residual interactions might be discarded in a first approximation. But there is no 'a priori' guarantee that this approximation is appropriate under all circumstances. This, in principle, should be examined by lattice QCD in order to test the cluster decomposition in hot QCD.

Related transition rates (to Eq. (7)) are defined for the fusion of three off-shell quarks $\left(q_{1}+q_{2}+q_{3} \leftrightarrow B\right)$ to a color neutral baryonic $(B$ or $\bar{B})$ resonances of finite width (or strings) fulfilling energy and momentum conservation as well as flavor current conservation, i.e.

$$
\begin{aligned}
& \frac{d N_{B}(x, p)}{d^{4} x d^{4} p}=T r_{q_{1}} \operatorname{Tr}_{q_{2}} \operatorname{Tr}_{q_{3}} \delta^{4}\left(p-p_{1}-p_{2}-p_{3}\right) \\
& \quad \delta^{4}\left(\frac{x_{q_{1}}+x_{q_{2}}+x_{q_{3}}}{3}-x\right) \\
& \quad \times \omega_{q_{1}} \rho_{q_{1}}\left(p_{1}\right) \omega_{q_{2}} \rho_{q_{2}}\left(p_{2}\right) \omega_{q_{3}} \rho_{q_{3}}\left(p_{3}\right) \\
& \quad \times\left|M_{q q q}\right|^{2} W_{B}\left(x_{1}, x_{2}, x_{3}, p_{1}, p_{2}, p_{3}\right) \\
& \quad \times N_{q_{1}}\left(x_{1}, p_{1}\right) N_{q_{2}}\left(x_{2}, p_{2}\right) N_{q_{3}}\left(x_{3}, p_{3}\right) \delta \text { (flavor, color). }
\end{aligned}
$$

Here the quantity $W_{B}$ denotes the baryon phase-space distribution (evaluated in Jacobi coordinates) which is taken again of Gaussian shape with a width of $1 \mathrm{fm}$ in coordinate space which corresponds to an average rms radius of excited baryons. The matrix element squared $\left|M_{q q q}\right|^{2}$ reflects the strength of three quark fusion processes and is fixed as follows: Since Regge trajectories for excited mesonic and baryonic states have the same slope (or string constant in the color dipole picture) we tentatively set $\left|M_{q q q}\right|^{2}=\left|v_{q \bar{q}}\right|^{3}$ in our present work which implies that (so far) there is no need to introduce any new parameter.

On the hadronic side PHSD includes explicitly the baryon octet and decouplet, the $0^{-}$and $1^{-}$meson nonets as well as selected higher resonances as in HSD [20]. Hadrons of higher masses $(>1.5 \mathrm{GeV}$ in case of baryons and $>1.3 \mathrm{GeV}$ in case of mesons) are treated as 'strings' (color-dipoles) that decay to the known (low mass) hadrons according to the JETSET algorithm [30].

\section{HADRONIZATION OF AN EXPANDING PARTONIC FIREBALL}

We now turn to actual results from PHSD for the model case of an expanding partonic fireball at initial temperature $T=1.7 T_{c}\left(T_{c}=0.185 \mathrm{GeV}\right)$ with quasiparticle properties and four-momentum distributions determined by the DQPM at temperature $T=1.7 T_{c}$.

\section{A. Initial conditions}

The initial distribution for quarks, antiquarks and gluons in coordinate space is taken as a Gaussian ellipsoid with a spatial eccentricity

$$
\epsilon=\left\langle y^{2}-x^{2}\right\rangle /\left\langle y^{2}+x^{2}\right\rangle
$$

and $\left\langle z^{2}>=<y^{2}>\right.$ in order to allow for the built-up of elliptic flow (as in semi-central nucleus-nucleus collisions at relativistic energies). In order to match the initial off-equilibrium strange quark content in relativistic $p p$ collisions the number of $s$ (and $\bar{s}$ quarks) is assumed to be suppressed by a factor of 3 relative to the abundance of $u$ and $d$ quarks and antiquarks. In this way we will be able to investigate additionally the question of strangeness equilibration. 
As mentioned above the dynamical evolution of the system is then entirely described by the transport dynamics in PHSD incorporating the off-shell propagation of the partonic quasiparticles according to Refs. [16] as well as the transition to resonant hadronic states (or 'strings') Eqs. (7), (9). The time integration for the testparticle equations of motion (cf. Refs. [16]) is performed in the same way as in case of hadronic off-shell transport where (in view of the momentum-independent width $\gamma$ ) the simple relation (19) in Ref. [31] is employed. For the collisions of partons two variants are at our disposal: i) geometrical collision criteria as employed in standard hadronic transport, ii) the in-cell method developed in Ref. [32]. The latter can easily be extended to describe $2 \leftrightarrow 3$ processes etc. in a covariant way [33] and is the better choice at high particle densities (cf. Ref. [34]). The hadronization is performed by integrating the rate equations (7) and (9) in space and time which are discretized by $\Delta t$ and $\Delta V(t)$. We use local cells of volume $d V(t)=0.25(1+b t)^{3} \mathrm{fm}^{3}$ where $t$ is given in units of $\mathrm{fm} / \mathrm{c}$ and $b=0.7 \mathrm{c} / \mathrm{fm}$. This choice approximately corresponds to a comoving grid for the expanding system. In each time-step $\Delta t$ and cell $\Delta V$ the integrals in (7) and (9) are evaluated by a sum over all (time-like) testparticles using (e.g. for the quark density)

$$
\begin{aligned}
& \frac{1}{\Delta V} \int_{\Delta V} d^{3} x \int \frac{d \omega_{q}}{2 \pi} \omega_{q} \int \frac{d^{3} p_{q}}{(2 \pi)^{3}} \rho_{q}\left(\omega_{q}, p_{q}\right) N_{q}\left(x, p_{q}\right) \\
& =\frac{1}{\Delta V} \sum_{J_{q} \varepsilon \Delta V} 1=\rho_{q}(\Delta V)
\end{aligned}
$$

where the sum over $J_{q}$ implies a sum over all testparticles of type $q$ (here quarks) in the local volume $\Delta V$ in each parallel run. In order to obtain lower fluctuations the integrals are averaged over the number of parallel runs (typically a few hundred). For each individual testparticle (i.e. $x_{q}$ and $p_{q}$ fixed) the additional integrations in (7) and (9) give a probability for a hadronization process to happen; the actual event then is selected by Monte Carlo. Since energy-momentum conservation fixes the four-momentum $p$ of the hadron produced - the spacetime position $x$ is fixed by (7) or (9) - the latter is represented by a hadronic state with flavor content fixed by the fusing quarks (antiquarks). The latter decays to the lower mass hadrons according to JETSET [30] above thresholds of $1.3 \mathrm{GeV}$ for mesonic states or $1.5 \mathrm{GeV}$ for baryonic states (as in HSD). Lower mass hadrons (octet and decouplet states) are determined by the weight of their respective spectral functions at given invariant mass and selected by Monte Carlo. Note that the propagation of partons includes the space-time derivatives of the quark and gluon mean-fields specified in Eq. (29) and displayed in Fig. 9 of Ref. [19].


FIG. 1: (Color online) Upper part: Time evolution of the total energy $E_{\text {tot }}$ (upper line), the partonic contributions from the interaction energy $V_{p}$ and the energy of time-like partons $T_{p}$ in comparison to the energy contribution from formed mesons $E_{m}$ and baryons (+ antibaryons) $E_{B+\bar{B}}$. Lower part: Time evolution in the parton, meson and baryon number for an expanding partonic fireball at initial temperature $T=1.7 T_{c}$ with initial eccentricity $\epsilon=0$.

\section{B. Dynamical evolution}

In Fig. 1 (upper part) we show the energy balance for the expanding system at initial temperature $T=1.7 T_{c}$ and eccentricity $\epsilon=0$, i.e. a fireball of spherical symmetry. The total energy $E_{\text {tot }}$ (upper line) - which at $t=0$ is given by (4) integrated over space - is conserved within $3 \%$ throughout the partonic expansion and hadronization phase such that for $t>8 \mathrm{fm} / \mathrm{c}$ it is given essentially by the energy contribution from mesons and baryons (+antibaryons). The initial energy splits into the partonic interaction energy $V_{p}$ (cf. Eq. (19) in Ref. [19]) and the energy of the time-like (propagating) partons

$$
T_{p}=\sum_{i} \sqrt{p_{i}^{2}+M_{i}^{2}\left(\rho_{p}\right)}
$$

with fractions determined by the DQPM [19]. In Eq. (12) the summation over $i$ runs over all testparticles in an individual run. The hadronization mainly proceeds during the time interval $1 \mathrm{fm} / \mathrm{c}<t<7 \mathrm{fm} / \mathrm{c}$ (cf. the lower part of Fig. 1 where the time evolution of the $q, \bar{q}, g$, meson and baryon (+antibaryon) number is displayed).

As one observes from Fig. 1 on average the number of hadrons from the resonance or 'string' decays is larger than the initial number of fusing partons. This might be 


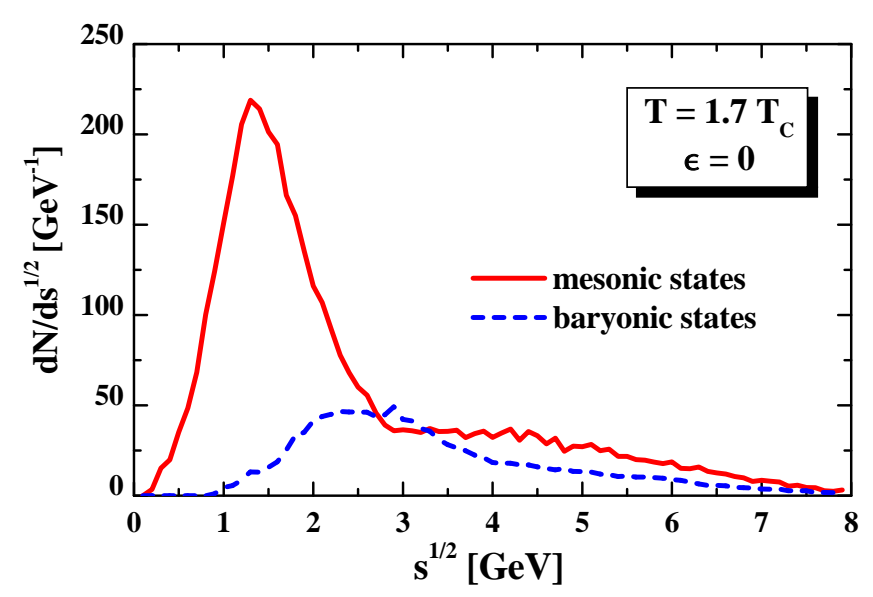

FIG. 2: (Color online) The invariant mass distribution for fusing $q \bar{q}$ pairs (solid line) as well as $q q q$ (and $\bar{q} \bar{q} \bar{q}$ ) triples (dashed line) that lead to the formation of final hadronic states for an expanding partonic fireball at initial temperature $T=1.7 T_{c}$ with initial eccentricity $\epsilon=0$.

astonishing since by partonic fusion the number of final states is conventionally reduced in coalescence models.

In order to shed some light on the hadronization process in PHSD we display in Fig. 2 the invariant mass distribution of $q \bar{q}$ pairs (solid line) as well as $q q q$ (and $\bar{q} \bar{q} \bar{q}$ ) triples (dashed line) that lead to the formation of final hadronic states. In fact, the distribution for the formation of baryon (antibaryon) states starts above the nucleon mass and extends to high invariant mass covering the nucleon resonance mass region as well as the high mass continuum (which is treated by the decay of strings within the JETSET model [30]). On the 'pre-mesonic side the invariant-mass distribution starts roughly above the two-pion mass and extends up to continuum states of high invariant mass (described again in terms of string excitations). The low mass sector is dominated by $\rho, a_{1}$, $\omega$ or $K^{*}, \bar{K}^{*}$ transitions etc. The excited 'pre-hadronic' states decay to two or more 'pseudoscalar octet' mesons such that the number of final hadrons is larger than the initial number of fusing partons.

Accordingly, the hadronization process in PHSD leads to an increase of the total entropy and not to a decrease as in case of coalescence models $[9,10]$. This is a direct consequence of the finite (and rather large) dynamical quark and antiquark masses as well as mean-field potentials which - by energy conservation - lead to 'prehadron' masses well above those for the pseudo-scalar meson octett or the baryon octett, respectively. This solves the entropy problem in hadronization in a natural way and is in accordance with the second law of thermodynamics!

The parton dynamics itself is governed by their propagation in the time-dependent mean-field $U_{p}\left(\rho_{p}\right)$ which is adopted in the parametrized form (as a function of the parton density $\rho_{p}$ ) given by Eq. (29) in Ref. [19]. Since the mean-field $U_{p}$ is repulsive the partons are accelerated



FIG. 3: (Color online) The effective gluon-gluon $\rightarrow$ gluongluon (solid line), gluon-quark $\rightarrow$ gluon-quark and quarkquark $\rightarrow$ quark-quark (dashed line) cross section from the DQPM as a function of the parton density $\rho_{p}$.

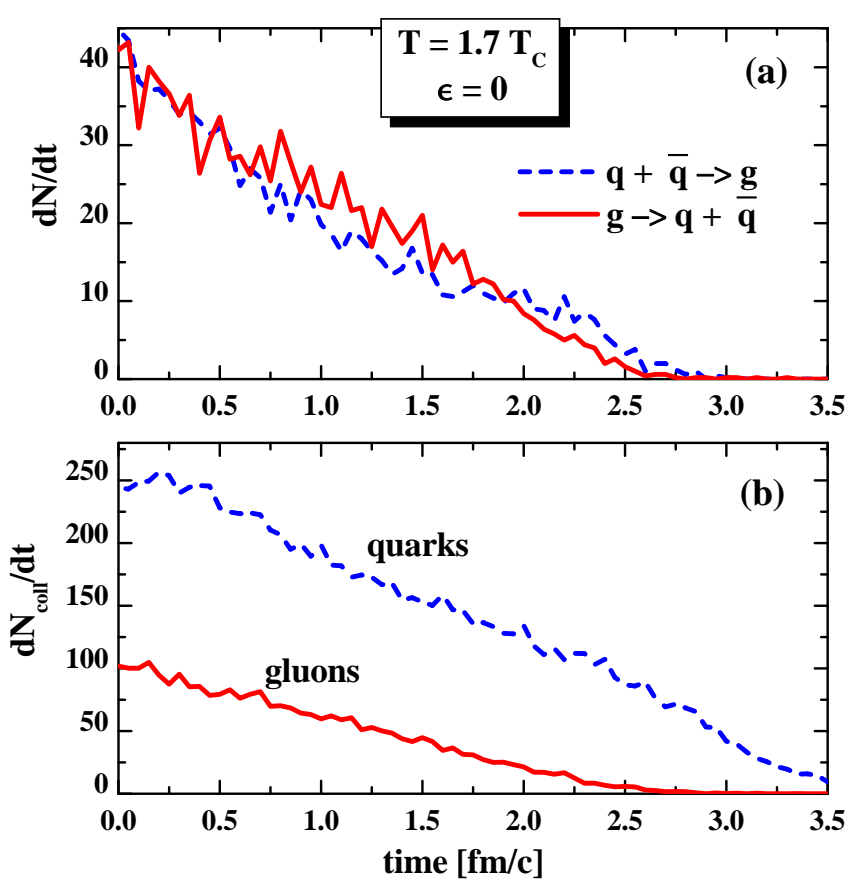

FIG. 4: (Color online) The interaction rate for the channels $q+\bar{q} \rightarrow g$ (dashed line, upper part) and $g \rightarrow q+\bar{q}$ (red line, upper part) for an expanding partonic fireball at initial temperature $T=1.7 T_{c}$ with initial eccentricity $\epsilon=0$. The lower part shows the collision rate of gluons (solid line) and quarks or antiquarks (dashed line).

during the expansion phase on expense of the potential energy density $V_{p}$ which is given by the integral of $U_{p}$ over $\rho_{p}$ (cf. Section 3 in Ref. [19]). The interaction rates of the partons are determined by effective cross sections which for $g g$ scattering have been determined in Ref. [4] as a function of $T / T_{c}$. The latter are re-parametrized in the actual calculation as a function of the parton density using the available dependence of $\rho_{p}(T)$ on the tempera- 

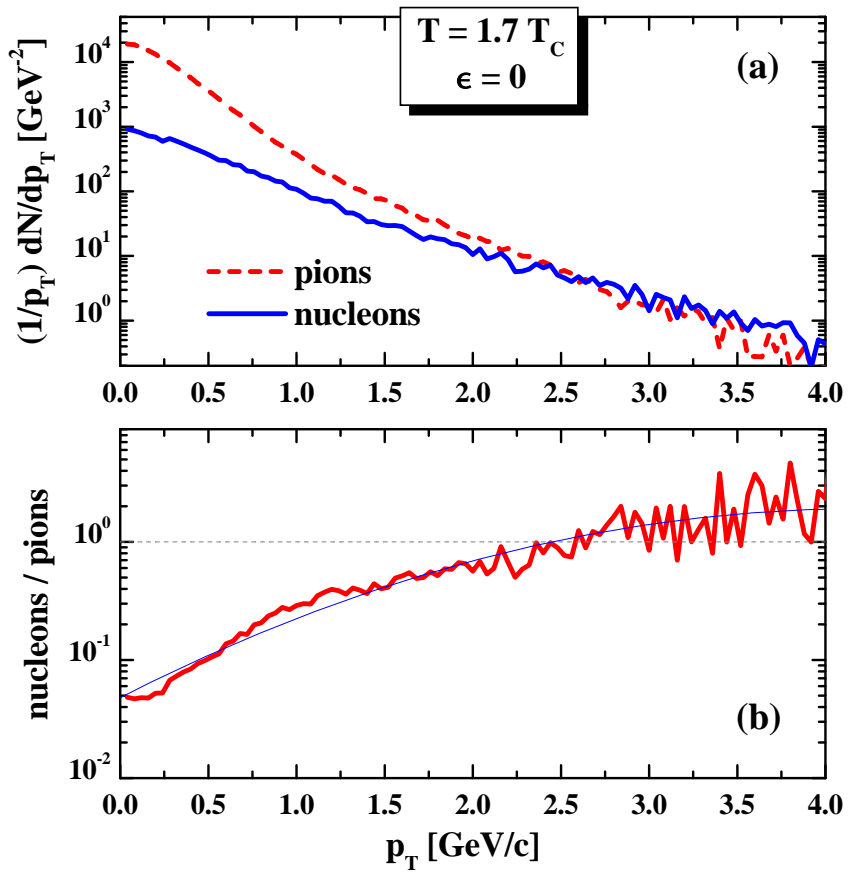

FIG. 5: (Color online) Upper part: The transverse momentum spectra $1 / p_{T} d N / d p_{T}$ for pions and nucleons for an expanding partonic fireball at initial temperature $T=1.7 T_{c}$ with initial eccentricity $\epsilon=0$. Lower part: The nucleon to pion ratio as a function of the transverse momentum $p_{T}$ corresponding to the spectra in the upper part. The thin blue line is drawn to guide the eye due to the limited statistics of the PHSD calculation.

ture $T$ from the DQPM.

The actual values for $g g$ scattering are shown in Fig. 3 as a function of the parton density $\rho_{p}$ (solid line) and demonstrate that $g g$ cross sections up to $20 \mathrm{mb}$ can be reached at $\rho_{p} \approx 2.5 \mathrm{fm}^{-3}$. The effective cross section drops rapidly with increasing $\rho_{p}$ which signals that weakly interacting partons might show up at very high parton density. Some note of caution has to be added here since though the cross section $\sigma_{g g}$ drops with $\rho_{p}$ the collision rate of a gluon $\left(\sim \sigma_{g g} \rho_{p}\right)$ increases slightly with $\rho_{p}$. For quark-quark or quark-antiquark elastic scattering the cross section is reduced by a factor $4 / 9$ in line with Casimir scaling. Quark-gluon elastic scattering (in the present implementation) is also reduced by a factor 4/9 (cf. Fig. 3, dashed line). The channels $q \bar{q} \rightarrow g$ are described by a relativistic Breit-Wigner cross section which is determined by the actual masses of the fermions, the invariant energy $\sqrt{s}$ and the resonance parameters of the gluon (from the DQPM). In this case a further constraint on flavor neutrality and open color is employed. The gluon decay to a $u \bar{u}, d \bar{d}$ or $s \bar{s}$ pair is fixed by detailed balance. Further channels are $g g \leftrightarrow g$ which are given by Breit-Wigner cross sections (with the gluon resonance parameters) and detailed balance, respectively.

The actual interaction rates for the channels $q+\bar{q} \rightarrow g$ and $g \rightarrow q+\bar{q}$ are displayed in Fig. 4 by the dashed line (upper part) and the solid line (upper part), respectively, for the expanding partonic fireball at initial temperature $T=1.7 T_{c}$ with initial eccentricity $\epsilon=0$. Within statistics the numerical result shows that detailed balance actually is fulfilled for the expanding partonic system which was initialized in thermal equilibrium. The lower part of Fig. 4 shows the total number of collisions per time for gluons (solid line) and quarks or antiquarks (dashed line) which is higher for the fermions since the latter are much more frequent than the gluons.

Let's, furthermore, have a look at the transverse momentum $p_{T}$ spectra of hadrons emerging from the PHSD dynamics of the expanding fireball initialized with $T=$ $1.7 T_{c}$. The resulting $p_{T}$ spectra are displayed in Fig. 5 (upper part) for pions and nucleons and show that the nucleons become more abundant for $p_{T}>2.5 \mathrm{GeV} / \mathrm{c}$. The ratio of nucleons to pions is depicted in the lower part of Fig. 5 and clearly demonstrate that baryons (antibaryons) become more frequent than mesons at high $p_{T}>2.5 \mathrm{GeV}$. This observation is in close analogy to the experimental findings in $\mathrm{Au}+\mathrm{Au}$ collisions at top RHIC energies [5]. Note that a quantitative comparison with RHIC data is not meaningful due to the rather simplified and special initial conditions employed here.

\section{Comparison to the statistical hadronization model}

It is, furthermore, interesting to have a look at the final particle ratios $K^{+} / \pi^{+}, p / \pi^{+}, \Lambda / K^{+}$etc. (after hadronic decays) which are shown in Table 1 . The latter ratios are compared to the grandcanonical statistical hadronization model (SM) [35-37] at baryon chemical potential $\mu_{B}=0$. For $\mu_{B}=0$ the particle ratios depend only on temperature $T$ and one may fix a freeze-out temperature, e.g., by the proton to $\pi^{+}$ratio. A respective comparison is given also in Table 1 for $T=160 \mathrm{MeV}$ and $170 \mathrm{MeV}$ for the SM which demonstrates that the results from PHSD are close to those from the SM for $T \approx 170 \mathrm{MeV}$. This also holds roughly for the $\Lambda / K^{+}$ratio. On the other hand the $K^{+} / \pi^{+}$ratio only smoothly depends on the temperature $T$ and measures the amount of strangeness equilibration. Recall that we initialized the system with a relative strangeness suppression factor of $1 / 3$. The deviation from the SM ratio by about $13 \%$ indicates that strangeness equilibration is not fully achieved in the calculations. This is expected since the partons in the surface of the fireball hadronize before chemical equilibration may occur. A detailed discussion of results will be presented in a forthcoming study.

The agreement between the PHSD and SM results for the baryon to meson ratio in the strangeness $S=0$ and $\mathrm{S}=1$ sector may be explained as follows: Since the final hadron formation dominantly proceeds via resonance and string formation and decay - which is approximately a microcanonical statistical process [38] - the average over many hadronization events with different energy/mass 


\begin{tabular}{|c|c|c|c|}
\hline & $p / \pi^{+}$ & $\Lambda / K^{+}$ & $K^{+} / \pi^{+}$ \\
\hline PHSD & 0.086 & 0.28 & 0.157 \\
\hline SM $T=160 \mathrm{MeV}$ & 0.073 & 0.22 & 0.179 \\
\hline SM $T=170 \mathrm{MeV}$ & 0.086 & 0.26 & 0.180 \\
\hline
\end{tabular}

TABLE I: Comparison of particle ratios from PHSD with the statistical model (SM) [37] for $T=160 \mathrm{MeV}$ and $170 \mathrm{MeV}$.

and particle number (in the initial and final state) leads to a grandcanonical ensemble. The latter (for $\mu_{B}=0$ ) is only characterized by the average energy or an associated Lagrange parameter $\beta=1 / T$.

\section{Elliptic flow}

Of additional interest are the collective properties of the partonic system during the early time evolution. In order to demonstrate the built up of elliptic flow we show in Fig. 6 the time evolution of

$$
v_{2}=\left\langle\left(p_{x}^{2}-p_{y}^{2}\right) /\left(p_{x}^{2}+p_{y}^{2}\right)\right\rangle
$$

for partons (solid line), mesons (long dashed line) and baryons (dot-dashed line) for an initial eccentricity $\epsilon=$ 0.33. As seen from Fig. 6 the partonic flow develops within $2 \mathrm{fm} / \mathrm{c}$ and the hadrons essentially pick up the collective flow from the accelerated partons. The hadron $v_{2}$ is smaller than the maximal parton $v_{2}$ since by parton fusion the average $v_{2}$ reduces and a fraction of hadrons is formed early at the surface of the fireball without a strong acceleration before hadronization. We briefly mention that the reduction of the average hadron elliptic flow essentially is due to the finite parton masses which are larger than the temperature in the hadronization phase. This reduced hadron $v_{2}$ is in contrast to the coalescence of massless partons. We discard a more detailed discussion and investigation of Eqs. (7) and (9) and refer the reader to an upcoming study.

It is important to point out that in PHSD the elliptic flow of partons predominantly stems from the gradients of the repulsive parton mean-fields (from the DQPM) at high parton (energy) density. To demonstrate this statement we show in Fig. 6 the result of a simulation without elastic partonic rescattering processes by the short dashed line.

Fig. 7 shows the final hadron $v_{2}$ versus the initial eccentricity $\epsilon$ and indicates that the ratio $v_{2} / \epsilon$ is practically constant $(\approx 0.2)$ as in ideal hydrodynamics (cf. Fig. 3 in Ref. [39]). Accordingly the parton dynamics in PHSD are close to ideal hydrodynamics. This result is expected since the ratio of the shear viscosity $\eta$ to the entropy density $s$ in the DQPM is on the level of $\eta / s \approx 0.2[4]$ and thus rather close to the lower bound of $\eta / s=1 /(4 \pi)$ [40]. Note that the ratio $\eta / s$ is dominantly determined by the quasiparticle width $\gamma(2)$ and low ratios on the level of $\eta / s \approx 0.2$ require broad parton spectral functions as employed in the DQPM.



FIG. 6: (Color online) Time evolution of the elliptic flow $v_{2}$ for partons and hadrons for the initial spatial eccentricity $\epsilon=$ 0.33 for an expanding partonic fireball at initial temperature $T=1.7 T_{c}$.

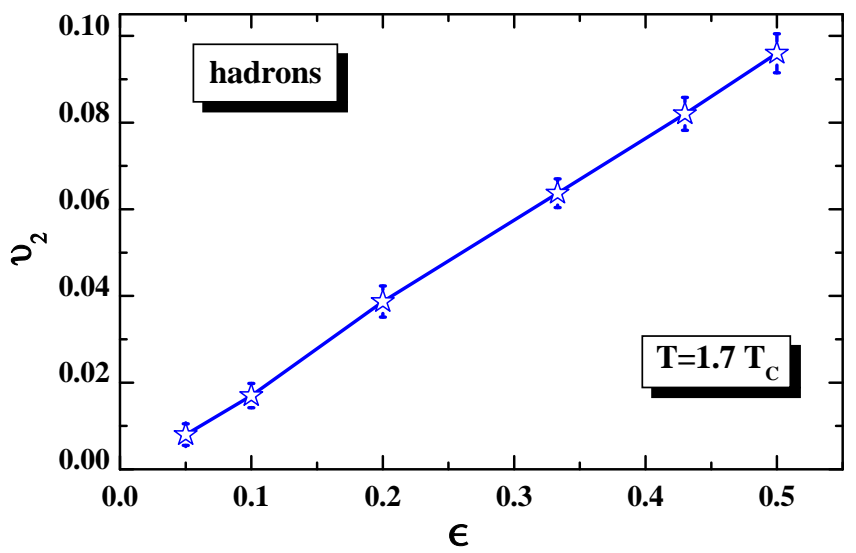

FIG. 7: (Color online) The hadron elliptic flow $v_{2}$ versus the initial spatial eccentricity $\epsilon$ for an expanding partonic fireball at initial temperature $T=1.7 T_{c}$.

A further test of the PHSD hadronization approach is provided by the 'constituent quark number scaling' of the elliptic flow $v_{2}$ which has been observed experimentally in central $\mathrm{Au}+\mathrm{Au}$ collisions at RHIC [5, 41]. In this respect we plot $v_{2} / n_{q}$ versus the transverse kinetic energy per constituent parton,

$$
T_{k i n}=\frac{m_{T}-m}{n_{q}},
$$

with $m_{T}$ and $m$ denoting the transverse mass and actual mass, respectively. For mesons we have $n_{q}=2$ and for baryons/antibaryons $n_{q}=3$. The results for the scaled elliptic flow are shown in Fig. 8 for mesons (dashed line) and baryons (solid line) and suggest an approximate scaling. We note that the scaled hadron elliptic flow $v_{2} / n_{q}$ does not reflect the parton $v_{2}$ at hadronization and is significantly smaller. Due to the limited statistics especially in the baryonic sector with increasing $p_{T}$ this issue will have to be re-addressed with high statistics in the actual 


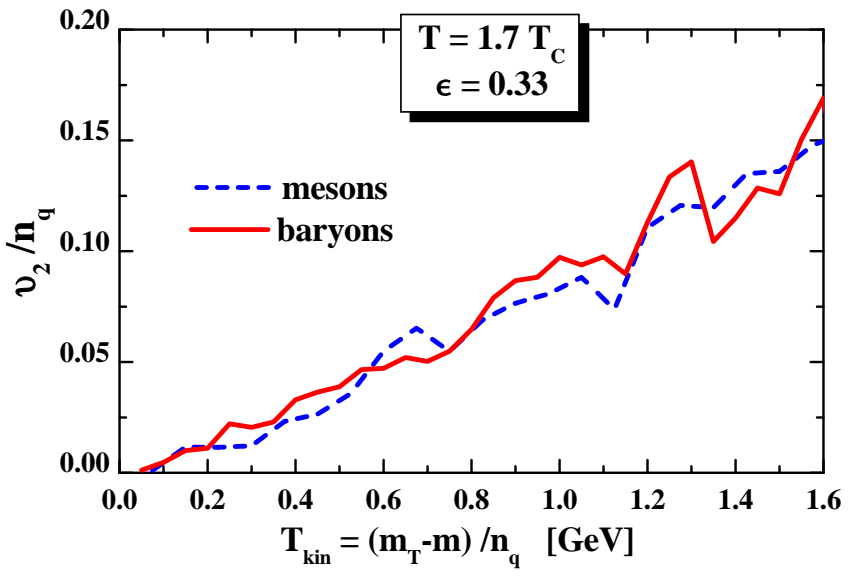

FIG. 8: (Color online) The elliptic flow $v_{2}$ - scaled by the number of constituent quarks $n_{q}$ - versus the transverse kinetic energy divided by $n_{q}$ for mesons (dashed line) and baryons (solid line).

heavy-ion case where the very early parton $p_{T}$ distribution also shows 'power-law' tails.

\section{SUMMARY}

In summary, the expansion dynamics of an anisotropic partonic fireball is studied within the PHSD approach which includes dynamical local transition rates from partons to hadrons (7), (9) (and vice versa). It shows collective features as expected from ideal hydrodynamics in case of strongly interacting systems. The hadronization process conserves four-momentum and all flavor currents and slightly increases the total entropy (by about $15 \%$ in the model case investigated here) since the 'fusion' of rather massive partons dominantly leads to the formation of color neutral strings or resonances that decay microcanonically to lower mass hadrons. This solves the entropy problem associated with the simple coalescence model!

We find that the hadron abundancies and baryon to meson ratios are compatible with those from the sta- tistical hadronization model $[35,36]$ - which describes well particle ratios from AGS to RHIC energies - at a freeze-out temperature of about $170 \mathrm{MeV}$. Furthermore, strangeness equilibration is approximately achieved in the dynamical expansion and driven by the processes $q \bar{q} \leftrightarrow g \leftrightarrow s \bar{s}$, which is a resonant process in the DQPM. However, although the final hadron ratios are compatible with a fixed freeze-out temperature $(\sim 170 \mathrm{MeV})$ we observe that the actual hadronization occurs at very different energy densities (or temperatures) (cf. also Ref. [42]) such that the microscopic studies do not support the sudden freeze-out picture.

Our calculations show that the hadron elliptic flow is essentially produced in the early partonic stage where also the strong repulsive parton mean-fields contribute to a large extent. This might explain why the hadron $v_{2}$ from HSD calculations [43] underestimated the RHIC data on $v_{2}$ essentially at midrapidity (in the pure hadron/string approach). The hadron elliptic flow from PHSD is smaller than the parton $v_{2}$ due to a partial cancellation of the $v_{2}$ values from the individual partons in the fusion process to hadrons (but larger than in HSD). This cancellation essentially is a consequence of parton masses that are larger than the 'local temperature' during the hadronization phase. Nevertheless, our observations indicate an approximate quark number scaling (cf. Fig. 8) for low and moderate transverse kinetic energies within the statistics reached so far. This issue will be followed up in more detail in a forthcoming investigation.

The present study, however, serves only as a model case which allows for a more transparent interpretation of the various results. An application of the PHSD approach to ultrarelativistic heavy-ion collisions especially in comparison to differential experimental data is expected to shed further light on the transport properties of the partonic phase and the dynamics of hadronization.

The authors like to thank A. Andronic for providing the SM results in Table 1 and for a critical reading of the manuscript. Furthermore, they are grateful to O. Linnyk for valuable discussions.
[1] Quark Matter 2005, Nucl. Phys. A 774 (2006) 1; Quark Matter 2006, J. Phys. G 34 (2007) S1.

[2] E. Shuryak, Prog. Part. Nucl. Phys. 53 (2004) 273.

[3] M. H. Thoma, J. Phys. G 31 (2005) L7.

[4] A. Peshier and W. Cassing, Phys. Rev. Lett. 94 (2005) 172301.

[5] I. Arsene et al., Nucl. Phys. A 757 (2005) 1; B. B. Back et al., Nucl. Phys. A 757 (2005) 28; J. Adams et al., Nucl. Phys. A 757 (2005) 102; K. Adcox et al., Nucl. Phys. A 757 (2005) 184.

[6] T. Hirano, M. Gyulassy, Nucl. Phys. A 769 (2006) 71.

[7] A. Ayala, M. Martinez, G. Paic, and G. Toledo Sanchez,
arXiv: 0710.3629 [hep-ph].

[8] S. Scherer et al., New J. Phys. 3 (2001) 8.

[9] R. C. Hwa and C. B. Yang, Phys. Rev. C 67 (2003) 034902; V. Greco, C. M. Ko and P. Levai, Phys. Rev. Lett. 90 (2003) 202302.

[10] R. J. Fries, B. Müller, C. Nonaka and S. A. Bass, Phys. Rev. Lett. 90 (2003) 202303.

[11] Z.-W. Lin et al., Phys. Rev. C 72 (2005) 064901.

[12] L. Ravagli, H. van Hees and R. Rapp, arXiv: 0806.2055 [hep-ph].

[13] T. S. Biro and K. Urmossy, Eur. Phys. J. ST 155 (2008) 1. 
[14] L. P. Kadanoff, G. Baym, Quantum Statistical Mechanics, Benjamin, 1962.

[15] S. Juchem, W. Cassing and C. Greiner, Phys. Rev. D 69 (2004) 025006; Nucl. Phys. A 743 (2004) 92.

[16] W. Cassing and S. Juchem, Nucl. Phys. A 665 (2000) 377; ibid A 672 (2000) 417.

[17] Y. B. Ivanov, J. Knoll and D. N. Voskresensky, Nucl. Phys. A 672 (2000) 313.

[18] W. Cassing, Nucl. Phys. A 791 (2007) 365.

[19] W. Cassing, Nucl. Phys. A 795 (2007) 70.

[20] W. Cassing and E. L. Bratkovskaya, Phys. Rep. 308 (1999) 65; W. Ehehalt and W. Cassing, Nucl. Phys. A 602 (1996) 449.

[21] A. Peshier, Phys. Rev. D 70 (2004) 034016; J. Phys. G 31 (2005) S371.

[22] O. Kaczmarek, F. Karsch, F. Zantow and P. Petreczky, Phys. Rev. D 70 (2004) 074505; ibid. D 72 (2005) 059903.

[23] R. D. Pisarski, Phys. Rev. Lett. 63 (1989) 1129.

[24] A. Peshier, B. Kämpfer, O.P. Pavlenko, and G. Soff, Phys. Rev. D 54 (1996) 2399; P. Levai and U. Heinz, Phys. Rev. C 57 (1998) 1879; A. Peshier, B. Kämpfer, and G. Soff, Phys. Rev. C 61 (2000) 045203, Phys. Rev. D 66 (2002) 094003.

[25] M. Bluhm, B. Kämpfer, R. Schulze, D. Seipt, and U. Heinz, Phys. Rev. C 76 (2007) 034901.

[26] J. Letessier and J. Rafelski, Phys. Rev. C 67 (2003) 031902.

[27] G. Aarts and J. M. Martinez Resco, J. High Energy Phys.
04 (2002) 054; ibid. 02 (2004) 030.

[28] C. B. Dover, U. Heinz, E. Schnedermann, and J. Zimanyi, Phys. Rev. C 44 (1991) 1636.

[29] S. J. Wang et al., Nucl. Phys. A 573 (1994) 245.

[30] H.-U. Bengtsson and T. Sjöstrand, Comp. Phys. Commun. 46 (1987) 43.

[31] E. L. Bratkovskaya and W. Cassing, Nucl. Phys A 807 (2008) 214.

[32] A. Lang, H. Babovsky, W. Cassing, U. Mosel, H.-G. Reusch, and K. Weber, J. of Comp. Phys. 106 (1993) 391.

[33] W. Cassing, Nucl. Phys. A 700 (2002) 618.

[34] Z. Xu and C. Greiner, Phys. Rev. C 71 (2005) 064901.

[35] P. Braun-Munzinger et al., Phys. Lett. B 365 (1996) 1; ibid. B 465 (1999) 15; ibid. B 518 (2001) 41.

[36] A. Andronic, P. Braun-Munzinger and J. Stachel, Nucl. Phys. A 772 (2006) 167.

[37] A. Andronic, private communication.

[38] F. Beccatini, Z. Phys. C 69 (1996) 485.

[39] S. A. Voloshin et al., J. Phys. G 34 (2007) S883.

[40] P. Kovtun, D. T. Son and A. O. Starinets, Phys. Rev. Lett. 94 (2005) 111601.

[41] B. I. Abelev et al., Pys. Rev. C 77 (2008) 054901.

[42] J. Knoll, arXiv:0803.2343 [nucl-th].

[43] E. L. Bratkovskaya, W. Cassing, and H. Stöcker, Phys. Rev. C 67 (2003) 054905; E. L. Bratkovskaya et al., Phys. Rev. C 69 (2004) 054907. 